$900{ }^{\circ} \mathrm{C}$

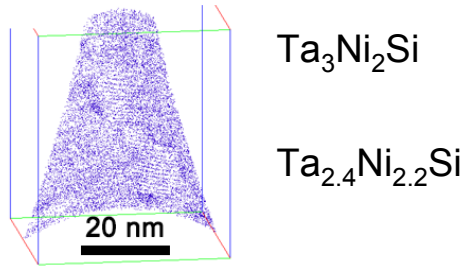

(C) 2015. This manuscript version is made available under the Elsevier user license http://www.elsevier.com/open-access/userlicense/1.0/ 


\title{
Atomic-Scale Structural Evolution of Ta-Ni-Si Amorphous Metal Thin Films
}

Richard P. Oleksak ${ }^{1}$, Arun Devaraj ${ }^{2}$, Gregory S. Herman ${ }^{1, *}$

${ }^{1}$ Oregon State University, School of Chemical, Biological and Environmental Engineering, Corvallis, OR 97331-2072, United States

${ }^{2}$ Environmental Molecular Sciences Laboratory, Pacific Northwest National Laboratory, P.O Box 999, Richland WA 99352.

\section{Corresponding Author}

*greg.herman@oregonstate.edu

\begin{abstract}
We investigated the thermal stability of a new ternary amorphous metal thin film, $\mathrm{Ta}_{2.4} \mathrm{Ni}_{2.2} \mathrm{Si}$, and assessed its suitability as a $\mathrm{Cu}$ diffusion barrier for semiconductor device applications. Transmission electron microscopy was coupled with atom probe tomography to provide a detailed understanding of the atomic-scale evolution of both structure and composition as a function of annealing temperature. We show that the amorphous structure is stable up to $>800{ }^{\circ} \mathrm{C}$ under ultrahigh vacuum, while annealing to $900{ }^{\circ} \mathrm{C}$ induces nano-crystallization of a single ternary phase in an amorphous matrix. The implications of crystallization and solute partitioning are examined in the context of high-temperature stability to aid in the design and understanding of this new class of thin film materials.
\end{abstract}

Key Words: amorphous metal thin film, transmission electron microscopy, atom probe tomography 


\section{Introduction}

As the feature size of integrated circuits continues to decrease, diffusion of $\mathrm{Cu}$ interconnect materials into silicon during device fabrication and operation is of increasing concern. Ta-based materials have shown particular promise as $\mathrm{Cu}$ diffusion barriers.[1] This is due to the refractory nature of Ta (resulting in low diffusivities), its low miscibility with $\mathrm{Cu}$, and its ability to form thermally stable amorphous phases that lack grain boundaries.[2] Pure Ta has been used[3] but is largely limited by the formation of polycrystalline films that result in grain boundary diffusion pathways. Amorphous materials such as Ta-N[1][3], Ta-C[4], Ta-Si[1] and Ta-Si-N[1] have shown improved barrier performance due primarily to high crystallization temperatures and thus lack of grain boundary diffusion pathways. Incorporation of Si into stable amorphous phases in the Ta-Si and Ta-Si-N systems has the added benefit of deterring diffusion of $\mathrm{Si}$ into the diffusion barrier and mitigating interfacial reactions. However, these improvements come at the expense of increased electrical resistivity that limits their widespread use.[5] Amorphous Ta-based alloys such as Ta-Ni[6], Ta-Co[7] and Ta-Fe[7] have reduced electrical resistivity but also suffer from depressed crystallization temperatures ranging from $<700{ }^{\circ} \mathrm{C}$ to $<800{ }^{\circ} \mathrm{C}$.

In light of the aforementioned trade-offs the ternary system of Ta-Ni-Si may be a promising candidate $\mathrm{Cu}$ diffusion barrier. Such a material combines the inherent advantages of Ta-based diffusion barriers described above with reduced electrical resistivity and resistance to interfacial reactions. Furthermore, the use of a multicomponent (i.e., ternary) system should allow for improved stability of the amorphous phase due to entropic considerations, thus inhibiting grain boundary formation to higher temperatures. By selecting the appropriate composition this system satisfies the design rules proposed by Inoue for formation of bulk 
metallic glasses (BMG).[8] In short, the material contains three components, is approximately 20 at\% metalloid $(\mathrm{Si})$, and the constituent atoms have large size variation and negative heats of mixing[9]. Despite the promising characteristics, this material has not been previously studied as a potential $\mathrm{Cu}$ diffusion barrier, and has only recently been investigated in the context of a thermally stable amorphous metal thin film (AMTF).[10] Hence in this study we investigated the thermal stability of sputter deposited AMFTs of approximate composition $\mathrm{Ta}_{2.4} \mathrm{Ni}_{2.2} \mathrm{Si}$. This composition satisfies the above design rules for creating metastable amorphous alloys and has demonstrated low electrical resistivity.[10] The films were annealed in ultrahigh vacuum and the structure and composition were monitored using transmission electron microscopy (TEM) and atom probe tomography (APT). APT is an excellent characterization technique capable of providing sub-nanometer scale spatially resolved compositional mapping of amorphous materials including thin films.[11][12][13][14][15] Together, these techniques allow observation of the atomic-scale evolution of structure and composition of this high temperature AMTF material.

\section{Experimental}

The films were deposited using RF magnetron sputtering from a single $\mathrm{Ta}_{2} \mathrm{Ni}_{2} \mathrm{Si}$ target (2 inch diameter, 0.25 inch thickness). Prior to deposition the chamber base pressure was $<10^{-6}$ Torr. A RF power of $100 \mathrm{~W}$, an Ar flow rate of 20 SCCM, and a chamber pressure of 5 mTorr were used during deposition. The resulting film composition was determined to be approximately $\mathrm{Ta}_{2.4} \mathrm{Ni}_{2.2} \mathrm{Si}$ by APT measurements, where $1-2$ at $\%$ oxygen was present in the films before and after annealing. The as-deposited films were annealed in an ultrahigh vacuum chamber (base pressure $\approx 2 \times 10^{-9}$ Torr) from room temperature to a specified temperature (800 or $900{ }^{\circ} \mathrm{C}$ ) using a $20^{\circ} \mathrm{C} / \mathrm{min}$ ramp rate and 30 min hold time. Transmission electron microscopy (TEM) analysis was performed using a spherical aberration corrected FEI Titan 80-300 TEM 
operating at $300 \mathrm{kV}$ or an FEI Titan 80-200 TEM operating at $200 \mathrm{kV}$. Atom probe tomography (APT) was performed using a CAMECA LEAP 4000XHR Atom probe tomography system using a pulsed UV laser (355 nm wavelength) with $20 \mathrm{pJ}$ laser pulse energy, and a 0.005 atoms/pulse evaporation rate during analysis with the sample temperature maintained at $40 \mathrm{~K}$. APT results were reconstructed and analyzed using IVAS 3.6.6 software. Samples for TEM and APT analysis were prepared using focused ion beam (FIB) based lift-out methods in an FEI Quanta 3D Dual Beam Scanning Electron Microscope (SEM).[16]

\section{Results and Discussion}

Cross-sectional bright field TEM imaging and associated selected area electron diffraction (SAED) patterns for an as-deposited Ta2.4Ni2.2Si film and the same film after annealing to 800 and $900{ }^{\circ} \mathrm{C}$ are shown in Fig. 1. 


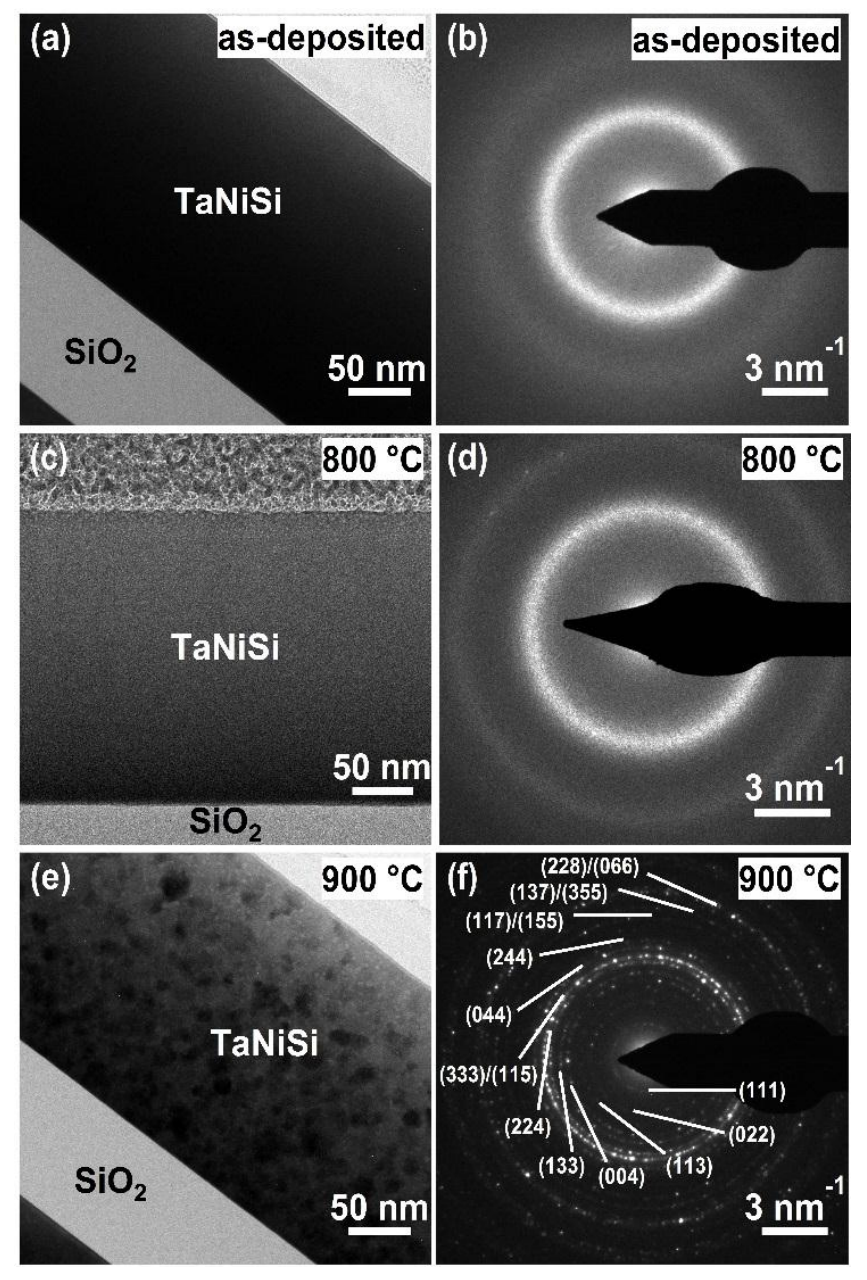

The as-deposited film (Fig. 1a) is uniform in appearance and smooth, demonstrating good conformality to the thermally oxidized Si substrate. Close inspection reveals a slight variation in contrast from the film surface to substrate and is attributed to thickness variation in the FIB cross-section resulting from the sample preparation process. SAED of the as-deposited film (Fig. 1b) shows two diffuse halos at 4.5 and $7.4 \mathrm{~nm}^{-1}$ corresponding to atomic spacings of 2.2 and 1.3 $\AA$, respectively. The lack of any discrete diffraction spots and the diffuse nature of the rings confirm the amorphous nature of the as-deposited films. No significant change is observed for films annealed up to $800{ }^{\circ} \mathrm{C}$ (Fig. 1c-d), while close inspection of the $800{ }^{\circ} \mathrm{C}$ SAED pattern (Fig. 1d) reveals a small number of faint diffraction spots near spacings of 2.2 and $1.3 \AA$. Thus after annealing to $800{ }^{\circ} \mathrm{C}$ the films remain largely amorphous, with some evidence of the very initial 
stages of crystallization. The crystallization temperature $\left(\mathrm{T}_{\mathrm{c}}\right)$ between 800 and $900{ }^{\circ} \mathrm{C}$ was further confirmed with in-situ heating TEM experiments (data not shown). This compares favorably to the binary Ta-based AMTFs described above[6][7][17] which all displayed $T_{c}$ below $800{ }^{\circ} \mathrm{C}$, and to the bulk ternary alloy of approximate composition $\mathrm{Ta}_{2} \mathrm{Ni}_{2} \mathrm{Co}$ with $\mathrm{T}_{\mathrm{c}}$ near $750{ }^{\circ} \mathrm{C}[18]$. Furthermore, the observed $\mathrm{T}_{\mathrm{c}}$ between 800 and $900{ }^{\circ} \mathrm{C}$ is approximately $200{ }^{\circ} \mathrm{C}$ higher than that reported for annealing $\mathrm{Ta}_{2} \mathrm{Ni}_{2} \mathrm{Si}$ at $20-50$ mTorr base pressure[10] suggesting impurities from the ambient may play a significant role in crystallization for this system.

For Ta $2.4 \mathrm{Ni}_{2.2} \mathrm{Si}$ films annealed to $900{ }^{\circ} \mathrm{C}$ (Fig. 1e) significant non-uniformities were observed, which resulted from devitrification. Dark regions ranging in size from approximately 5 to $50 \mathrm{~nm}$ are observed throughout the film suggesting formation of regions with higher electron density or diffraction contrast due to crystallization in the amorphous matrix. The corresponding SAED pattern (Fig. 1f) confirms the film has crystallized, where diffraction spots and rings suggest the film consists of a distinct nano-crystalline phase rather than a mixture of nanocrystalline phases. This analysis determined that the material crystallized into a single ternary phase of cubic $\mathrm{Ta}_{3} \mathrm{Ni}_{2} \mathrm{Si}$ with no binary intermediates. The corresponding SAED pattern (Fig. 1d) can be exclusively indexed to this structure, and is consistent with X-ray diffraction data.[19] No significant reaction between the $\mathrm{Ta}_{2.4} \mathrm{Ni}_{2.2} \mathrm{Si}$ film and the thermal silicon oxide is seen for temperatures as high as $900{ }^{\circ} \mathrm{C}$.

To gain insight into the crystallization process a more detailed TEM analysis was conducted on the film annealed to $900{ }^{\circ} \mathrm{C}$ and is shown in Fig. 2 . 


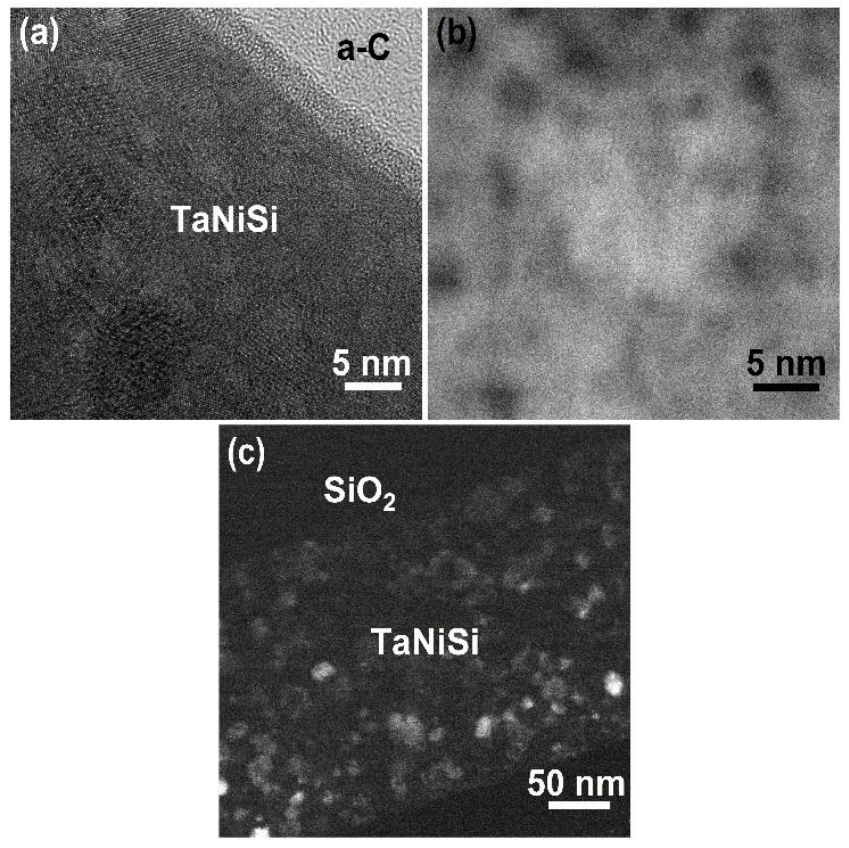

A bright-field HRTEM image near the film surface (Fig. 2a) reveals multiple crystalline domains dispersed in largely amorphous regions and a surface oxide $\approx 3 \mathrm{~nm}$ in thickness. This surface oxide is also present in the as-deposited films, suggesting no significant segregation of oxygen impurities to the film surface or into the bulk of the film occurred during annealing. The film surface remains very smooth up to $900{ }^{\circ} \mathrm{C}$ even after crystallization, which may be important for device integration.[20][21] In addition to darker crystalline regions, sub-5 $\mathrm{nm}$ regions of lighter contrast are observed dispersed throughout the film. A high-angle annular dark-field (HAADF) image of the $900{ }^{\circ} \mathrm{C}$ annealed film is shown in Fig. $2 \mathrm{~b}$. A crystalline region $\approx 15 \mathrm{~nm}$ in diameter is observed in the center of the image. The lighter contrast of this region is consistent with the increased scattering from the Ta-rich $\mathrm{Ta}_{3} \mathrm{Ni}_{2} \mathrm{Si}$ phase. Similar to the bright field image (Fig. 2a), periodic sub 5-nm regions of low electron density are observed, which appear dark in the HAADF image. These regions may correspond to the segregation of oxygen impurities and/or the formation of Ni- or Si-rich clusters during the solute partitioning which accompanies crystallization of the Ta-rich phase. Although these high-resolution images provide insight to the 
structure of crystallized regions, the nature of the cross-sectional sample makes it difficult to understand the size and distribution of crystallites as multiple crystal domains throughout the sample thickness contribute to image formation. To reduce the impact of sample thickness, centered dark field imaging (CDF) was used (Fig. 2c). This diffraction contrast image was acquired using approximately 5 to $10 \%$ of the total SAED spots corresponding to $\mathrm{Ta}_{3} \mathrm{Ni}_{2} \mathrm{Si}$ diffraction features in the range of 2.0 to $2.3 \AA$ and thus represents a significantly smaller fraction of the total $\mathrm{Ta}_{3} \mathrm{Ni}_{2} \mathrm{Si}$ regions. A broad distribution of light contrast crystalline regions are observed in this CDF image ranging from approximately $50 \mathrm{~nm}$ to sub-5 $\mathrm{nm}$. These regimes are seen to exist relatively uniformly throughout the film thickness, with no significant difference in concentration near the film surface or substrate interface.

To gain insight into the nanoscale composition variation accompanying the structural evolution described above, APT analysis was performed for films after each annealing condition and the results are summarized in Fig. 3.
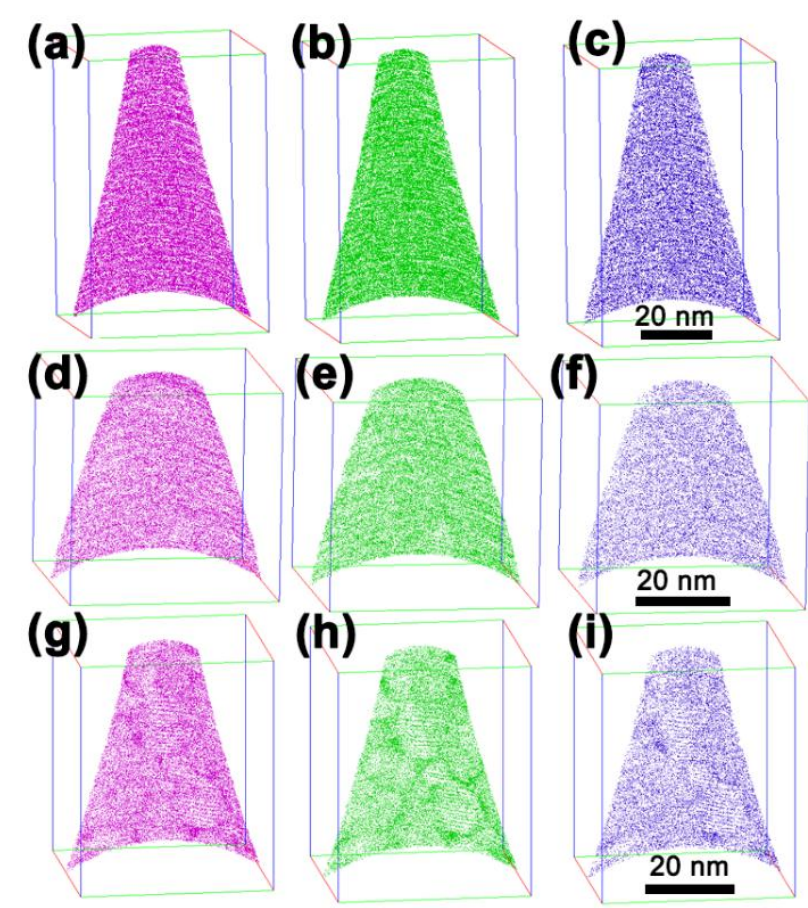
From left to right we show the individual ion maps for Ta, Ni and Si. The APT data from asdeposited (Fig. 3a-c) and $800{ }^{\circ} \mathrm{C}$ films (Fig. 3d-f) are seen to contain a uniform distribution of metal ions, consistent with the amorphous structure observed by TEM (Fig. 1a-d). Close inspection of the $900{ }^{\circ} \mathrm{C}$ film (Fig. 3j-i) reveals significant non-uniformity accompanying the devitrification process. A detailed analysis of the $900{ }^{\circ} \mathrm{C}$ film is shown in Fig. 4 .
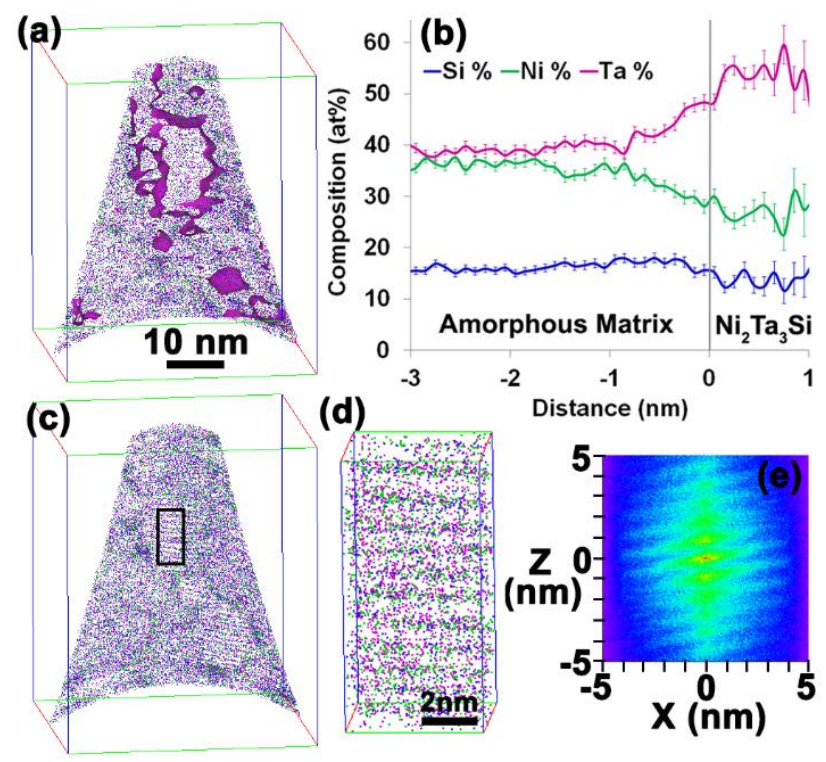

It was found that the observed non-uniformities corresponded to Ta-rich regions. A $3 \mathrm{~nm}$ slice of the APT reconstruction of $\mathrm{Ta}, \mathrm{Ni}$ and $\mathrm{Si}$ ions is shown in Fig. 4a, where an isocomposition surface showing regions containing $>42$ at $\%$ Ta is highlighted as opaque regions. A wide distribution of sizes is observed for these regions ranging from approximately 2 to $20 \mathrm{~nm}$. A proximity histogram showing the relative at\% of each metal across an isocomposition surface is shown in Fig. 4b, where 0 marks the interface of a Ta-rich region and surrounding matrix. A clear transition is observed over a distance of $\approx 1 \mathrm{~nm}$, where the relative at $\% \mathrm{Ta}: \mathrm{Ni}: \mathrm{Si}$ changes from approximately 2.4:2.2:1 to 3:2:1 indicating the Ta-rich regions have composition $\mathrm{Ta}_{3} \mathrm{Ni}_{2} \mathrm{Si}$. This composition is consistent with the structure determined from diffraction measurements (Fig. 
1f) suggesting the Ta-enrichment observed in APT analysis corresponds to crystallized regions of cubic $\mathrm{Ta}_{3} \mathrm{Ni}_{2} \mathrm{Si}$. This suggests a direct crystallization process in the Ta-Ni-Si system, despite the existence of several stable binary phases. This apparent suppression of binary phase formation may be related to the exceptionally high crystallization temperature observed for this system. Another $3 \mathrm{~nm}$ slice of the ion maps of Ni, Ta and Si is shown in Fig. 4c. In addition to general non-uniformities in the spatial distribution of $\mathrm{Ni}, \mathrm{Ta}$, and $\mathrm{Si}$, a region of long-range order $(\approx 10 \mathrm{~nm})$ is observed in the same area for each metal (highlighted with the black rectangle). A higher resolution image of this region showing a $5 \times 5 \times 10 \mathrm{~nm}^{3}$ reconstruction is shown in Figure 4d. Approximately 10 atomic planes are clearly observed, with an average spacing of $\approx 1.1 \mathrm{~nm}$. A Z-X spatial distribution map[22] plotted in the intermetallic region (Figure 4e) also clearly illustrates the $1.1 \mathrm{~nm}$ spacing of the observed atomic planes. This spacing is consistent with the lattice constant of cubic $\mathrm{Ta}_{3} \mathrm{Ni}_{2} \mathrm{Si}$. Thus, APT analysis allows for the direct observation of (001) planes in crystallized regions surrounded by an amorphous matrix.

\section{Conclusions}

The temperature dependent structure and composition of Ta-Ni-Si thin films was monitored at the near atomic-scale using state of the art TEM and APT techniques. The high glass forming ability of this system largely suppresses the formation of unary and binary phases, with crystallization resulting in discrete nano-crystalline domains of a single ternary phase, $\mathrm{Ta}_{3} \mathrm{Ni}_{2} \mathrm{Si}$. The exceptional thermal stability suggests this material may be a promising candidate for Ta-based $\mathrm{Cu}$ diffusion barriers and other high temperature applications.

\section{Acknowledgements}

R. P. O. acknowledges support from the Center for Sustainable Materials Chemistry, which is supported by the U.S. National Science Foundation under Grant CHE-1102637. G. S. 
H. acknowledges support from the Semiconductor Research Corporation under contract number 2013-OJ-2438.001. The authors thank Nick Landau and Brendan Flynn for performing film deposition and annealing, respectively, Kris Olsen for performing preliminary X-ray diffraction measurements, and John McGlone, John Wager, and Doug Keszler for valuable discussions. The atom probe tomography experiments in this study were supported by the science theme user proposal funding (Proposal \# 47950) from William R. Wiley Environmental Molecular Sciences Laboratory (EMSL), a national scientific user facility sponsored by DOE's Office of Biological and Environmental Research located at PNNL. PNNL is operated by Battelle for the DOE under Contract DE-AC05-76RLO1830.

\section{References}

[1] Kolawa E, Chen JS, Reid JS, Pokela PJ, Nicolet MA. Tantalum-Based Diffusion-Barriers in Si/Cu VLSI Metallizations. J Appl Phys 1991;70:1369-73. doi:10.1063/1.349594.

[2] Kaloyeros AE, Eisenbraun E. Ultrathin diffusion barriers/liners for gigascale copper metallization. Annu Rev Mater Sci 2000;30:363-85. doi:10.1146/annurev.matsci.30.1.363.

[3] Holloway K, Fryer PM, Cabral C, Harper JME, Bailey PJ, Kelleher KH. Tantalum as a Diffusion Barrier Between Copper and Silicon - Failure Mechanism and Effect of Nitrogen Additions. J Appl Phys 1992;71:5433-44. doi:10.1063/1.350566.

[4] Tsai HY, Sun SC, Wang SJ. Characterization of sputtered tantalum carbide barrier layer for copper metallization. J Electrochem Soc 2000;147:2766-72. doi:10.1149/1.1393604.

[5] Wong HY, Shukor NFM, Amin N. Prospective development in diffusion barrier layers for copper metallization in LSI. Microelectronics J 2007;38:777-82. doi:10.1016/j.mejo.2007.04.011.

[6] Fang JS, Hsu TP, Chen GS. Crystallization and failure behavior of Ta-Ni nanostructured/amorphous diffusion barriers for copper metallization. J Electron Mater 2004;33:1176-81. doi:10.1007/s11664-004-0120-z. 
[7] Fang JS, Hsu TP, Chen GS. Crystallization and failure behavior of Ta-TM (TM = Fe, Co) nanostructured/amorphous diffusion barriers for copper metallization. J Electron Mater 2006;35:15-21. doi:10.1007/s11664-006-0178-x.

[8] Suryanarayana C, Inoue A. Bulk Metallic Glasses. Boca Raton, FL: CRC Press; 2011.

[9] Takeuchi A, Inoue A. Calculations of mixing enthalpy and mismatch entropy for ternary amorphous alloys. Mater Trans Jim 2000;41:1372-8.

[10] McGlone JM, Olsen KR, Stickle WF, Abbott JE, Pugliese RA, Long GS, et al. Ta-Based Amorphous Metal Thin Films. J Alloys Compd 2015. doi:10.1016/j.jallcom.2015.07.226.

[11] Nag S, Mahdak KC, Devaraj A, Gohil S, Ayyub P, Banerjee R. Phase separation in immiscible silver-copper alloy thin films. J Mater Sci 2009;44:3393-401. doi:10.1007/s10853-009-3449-0.

[12] Katakam S, Devaraj A, Bowden M, Santhanakrishnan S, Smith C, Ramanujan R V., et al. Laser assisted crystallization of ferromagnetic amorphous ribbons: A multimodal characterization and thermal model study. J Appl Phys 2013;114:184901. doi: $10.1063 / 1.4829279$.

[13] Puthucode A, Devaraj A, Nag S, Bose S, Ayyub P, Kaufman MJ, et al. De-vitrification of nanoscale phase-separated amorphous thin films in the immiscible copper-niobium system. Philos Mag 2014;94:1622-41. doi:10.1080/14786435.2014.892223.

[14] DeGeorge V, Devaraj A, Keylin V, Cui J, McHenry ME. Mass Balance and Atom Probe Tomography Characterization of Soft Magnetic ( $\left.\mathrm{Fe}_{65} \mathrm{CO}_{665}\right){ }_{79.5} \mathrm{~B}_{13} \mathrm{Si}_{2} \mathrm{Nb}_{4} \mathrm{Cu}_{1.5}$ Nanocomposites. IEEE Trans Magn 2015;51:1-4. doi:10.1109/TMAG.2014.2373333.

[15] Chen MW, Inoue A, Sakurai T, Ping DH, Hono K. Impurity oxygen redistribution in a nanocrystallized $\mathrm{Zr}(65) \operatorname{Cr}(15) \mathrm{Al}(10) \operatorname{Pd}(10)$ metallic glass. Appl Phys Lett 1999;74:812-4. doi:10.1063/1.123376.

[16] Thompson K, Lawrence D, Larson DJ, Olson JD, Kelly TF, Gorman B. In situ sitespecific specimen preparation for atom probe tomography. Ultramicroscopy 2007;107:131-9. doi:10.1016/j.ultramic.2006.06.008.

[17] Yan H, Tay YY, Liang MH, Chen Z, Ng CM, Pan JS, et al. Amorphous metallic thin films as copper diffusion barrier for advanced interconnect applications. 2009 11th Electron. Packag. Technol. Conf., IEEE; 2009, p. 567-72. doi:10.1109/EPTC.2009.5416485.

[18] Meng D, Yi J, Zhao DQ, Ding DW, Bai HY, Pan MX, et al. Tantalum based bulk metallic glasses. J Non Cryst Solids 2011;357:1787-90. doi:10.1016/j.jnoncrysol.2011.01.020.

[19] Gladishevs'kii E. Crystal structures of compounds of two transition metals with silicon and phase equilibriums in their triple systems. Porosk Metall 1962;2:4. 
[20] Chu JP, Jang JSC, Huang JC, Chou HS, Yang Y, Ye JC, et al. Thin film metallic glasses: Unique properties and potential applications. Thin Solid Films 2012;520:5097-122. doi:10.1016/j.tsf.2012.03.092.

[21] Cowell EW, Alimardani N, Knutson CC, Conley JF, Keszler DA, Gibbons BJ, et al. Advancing MIM electronics: amorphous metal electrodes. Adv Mater 2011;23:74-8. doi:10.1002/adma.201002678.

[22] Geiser BP, Kelly TF, Larson DJ, Schneir J, Roberts JP. Spatial distribution maps for atom probe tomography. Microsc Microanal 2007;13:437-47. doi:10.1017/S1431927607070948.

\section{Figure Captions}

Fig. 1. Bright-field TEM images and corresponding SAED patterns of Ta2.4Ni2.2Si films asdeposited (a, b), and after annealing to $800{ }^{\circ} \mathrm{C}(\mathrm{c}, \mathrm{d})$ and $900{ }^{\circ} \mathrm{C}(\mathrm{e}, \mathrm{f})$.

Fig. 2. TEM imaging of Ta2.4Ni2.2Si film annealed to $900{ }^{\circ} \mathrm{C}$. BFTEM (a), HAADF (b) and CDFTEM (c).

Fig. 3. APT results of $\mathrm{Ta}_{2 .} \mathrm{Ni}_{2.2} \mathrm{Si}$ films showing a $3 \mathrm{~nm}$ slice of the ion maps of $\mathrm{Ta}, \mathrm{Ni}$ and $\mathrm{Si}$ in as-deposited (a-c), $800{ }^{\circ} \mathrm{C}(\mathrm{d}-\mathrm{f})$ and $900{ }^{\circ} \mathrm{C}$ (g-i) samples.

Fig. 4. Detailed APT analysis of Ta2.4Ni2.2Si film annealed to $900{ }^{\circ} \mathrm{C}$. APT reconstruction of Ta, $\mathrm{Ni}$ and Si ions, with opaque isocompositional regions with > 42at\% Ta (a), proximity histogram showing relative at\% of each metal across isocomposition surface (b), APT reconstruction of $\mathrm{Ta}, \mathrm{Ni}$ and $\mathrm{Si}$ ions with region of long-range order highlighted (c), higher resolution image of highlighted region (d), and Z-X distribution map of highlighted region (e). 